PROCEEDINGS ARTICLE

\title{
Robotic Services for New Paradigm Smart Cities Based on Decentralized Technologies
}

\author{
Aleksandr Kapitonov, ${ }^{* \dagger}$ Sergey Lonshakov, ${ }^{\ddagger}$ Ivan Berman, ${ }^{\S}$ Eduardo Castelló Ferrer, ${ }^{\text {qI }}$ \\ Fabio P. Bonsignorio ${ }^{\dagger \dagger}$ Vitaly Bulatov, ${ }^{\ddagger}$ Aleksandr Svistov ${ }^{\S \S}$
}

\begin{abstract}
This article describes different methods of organizing robotic services for smart cities using secure encrypted decentralized technologies and market mechanisms-as opposed to models based on centralized solutions based (or not) on using cloud services and stripping citizens of the control of their own data. The basis of the proposed methods is the Ethereum decentralized computer with the mechanism of smart contracts. In this work, special attention is paid to the integration of technical and economic information into one network of transactions, which allows creating a unified way of interaction between robotsthe robot economy. Three possible scenarios of robotic services for smart cities based on the economy of robots are presented: unmanned aerial vehicles (UAVs), environmental monitoring, and smart factories. In order to demonstrate the feasibility of the proposed scenarios, three experiments are presented and discussed. Our work shows that the Ethereum network can provide, through smart contracts and their ability to activate programs to interact with the physical world, an effective and practical way to manage robot services for smart cities.
\end{abstract}

\section{Introduction}

In recent years, the demand for robotic devices for business and for personal use has significantly increased. According to various forecasts, the number of devices in the world market of Internet of Things (IoT) will be 30 billion units by $2020,{ }^{1}$ and by 2025 the number of connected IoT devices will grow to 75 billion. That said, in 2019, 35\% of companies in the field of logistics, public health, utilities, and resource management will resort to robotics solutions of some kind. ${ }^{2}$

Robotization of our economy and society will ultimately lead to the spread of (worldwide connected) smart cities: the integration of information and communications technologies and the IoT for the management of the city and its environment. Currently, many projects have already been launched to implement these ideas (e.g., Barcelona, Neom, and Amsterdam), ${ }^{3-5}$ so it is not surprising that many scientists and developers joined forces to solve the issue of the rational

\footnotetext{
* 0x3c71D90CC3f19aC14C5E59047bb1EcD6D3527D71

$\dagger$ A. Kapitonov (kapitonov.aleksandr@corp.ifmo.ru) is Associate Professor at ITMO University, Saint Petersburg, Russia.

$\ddagger$ S. Lonshakov (research@aira.life) is founder of Airalab, Tolyatti, Russia.

$\S$ I. Berman (ivan@dczd.tech) is Consulting Analyst at Drone Employee, San Francisco, USA.

II E. Castelló Ferrer (ecstll@media.mit.edu) is post-doctoral researcher at MIT Media Lab, MIT, Cambridge, USA.

$\dagger$ F. P. Bonsignorio (fabio.bonsignorio@sssup.it) is Professor at the BioRobotics Institute, Scuola Superiore Sant'Anna, Pisa and also CEO of Heron Robots, Genova, Italy.

$\$$ V. Bulatov (vitl@dczd.tech) is COO at Drone Employee, San Francisco, USA.

$\S \S$ A. Svistov (dev@drone-employee.com) is Engineer at Drone Employee, San Francisco, USA.
} 
organization of smart cities. An increasing amount of technologies are being added to the field of smart urban management, and new solutions for the organization and commercialization of their work are being sought. ${ }^{6,7}$

However, before such ambitious projects can be undertaken, large-scale problems of creation of giant multi-agent networks of cyber-physical systems (CPSs) must be solved. Firstly, a large number of connected devices lead to more demanding safety requirements. ${ }^{8}$ This leads many times to models of management that centralize both the information processes and, more critically, data ownership. Many times the cities sub-contract those services to big global operators who profit from the citizens' data. Therefore there are serious reasons to look for more open, multi-vendor market-oriented solutions allowing citizens to keep the ownership of their data. Multi-vendor architectures, because of the wide variety of robotic devices (static sensors, drones, mobile robots, unmanned vehicles, etc.), face serious challenges due to the networks' heterogeneity. ${ }^{9}$ This raises the need for developing adequate standards and architectures. ${ }^{10}$ More critically, the scalability of the network-measured by the number of unique devices, computational requirements, and amount of data - needs to be ensured.

Privacy of the citizens' data in the city becomes the main issue. ${ }^{11,12}$ The problem of registering devices and checking their activity affects the state, public and legal services. ${ }^{9,13}$ Compliance with the legislation needs to be enforced by design. For business, the key issue of introducing CPSs to the market is to create value and make profit from their activities, and for this it is necessary to guarantee coordination of economic and technical transactions within one secure and distributed network.

These questions force researchers to search for technologies that can solve the problems of multi-agent systems for a smart city and to satisfy society's demands.

In this article, we will present our vision of a new paradigm in the organization of robotic services for smart cities, which guarantees by design the efficiency and effectiveness of market processes and the privacy and data ownership of citizens. We will first explain why decentralized technologies and market mechanisms are suitable for resolving organizational problems (Section 2). Next, we will present concrete concepts of services, whose work can be organized according to these principles: unmanned transport systems (Section 3), environmental monitoring (Section 4), and production services (Section 5). In Section 6, we give specific examples of experiments concerning the concepts described.

\section{Decentralized Technologies and Robot Economy}

Nowadays, when the organization and coordination of the work of many robotic devices is needed, it is common to see a centralized ${ }^{14}$ approach, in which, as a rule, the data processing center concentrates the data and the management of the processes. However, as the network grows, the disadvantages of this approach begin to appear: as the number of information transactions increases, the load on the center grows. To maintain capacity, it is necessary to always increase it. The center is exposed to the risk of hacking (e.g., Equifax, Facebook, etc.), and the entire network is exposed to risk due to a malfunction of the center. Ultimately, researchers come to the conclusion that in order to create a large-scale structure of millions of CPSs, such as a smart city, decentralized management of them is necessary. ${ }^{15-17}$

Blockchains are an actual example of how a decentralized technology can find applications in 
various fields. Researchers have already shown the possibility of using them to create a network of robotic devices, ${ }^{18,19}$ and their ability to solve problems in the multi-agent network of CPSs is in many ways more effective than the current centralized methods of organization. ${ }^{14}$

One of the most important consequences of this technology was the emergence of the distributed Ethereum computing platform, whose nodes are able to conclude and execute smart contracts - protected software algorithms that are performed depending on the conditions prescribed. ${ }^{20}$ We believe that this technology is potentially capable of becoming the main key to the organization of smart cities.

Firstly, the distributed computer inherits all the advantages of blockchain technology: information logging; the ability to organize data privacy while preserving the publicity of relations between network agents; and fault tolerance against faults of individual agents. ${ }^{21}$ This already covers the need for security and privacy, and also provides a mechanism for creating a register of their activities for state regulators.

Secondly, a combination of the program logic of robotic devices and automatic economic transactions became possible for the first time with Ethereum. This opened the way for the market mechanism for organizing the work of the network of agents-the economy of robots or robonomics. ${ }^{22,23}$ In this structure, each agent is endowed with the ability to make deals for digital money (Fig. 1).

Robonomics offers significant advantages for the organization of smart cities. The work of autonomous agents will be subject to the laws of the market, where the methods of game theory, decision theory, and economic mechanisms are applicable. ${ }^{24,25}$ This allows solving problems common to agents in an optimal way. The robonomics method is equally applicable to any type and number of agents, so long as there is a way to connect them to a decentralized computer. As a standard solution for agent management, we suggest using the Robot Operating System (ROS) framework. ${ }^{26}$ This solves the problem of heterogeneity and allows creating a single standard for interaction between devices.

The main obstacle to the full implementation of a decentralized computer is the scalability of the Ethereum network and the speed of transaction processing. ${ }^{27}$ At present there are an average of 15 transactions per second - this is not enough for a network of millions of agents. ${ }^{28}$ The reason for this low speed lies in the mechanism for reaching a consensus between Proof-of-work nodes, which depends on the computing power of the nodes. Developers of the platform are already dealing with this problem: a sharding mechanism was proposed, in which it is proposed to divide the network data into several parts and distribute it to different servers. ${ }^{27}$ The transition to hybrid consensus schemes that combine the Proof-of-stake mechanisms (based on the monetary balance of the nodes) and the Proof-of-authority (the availability of approved transaction-validating nodes) will also significantly speed up transaction confirmation. ${ }^{29,30}$ Developers are expected to achieve 1 million transactions per second. ${ }^{31}$

An important consequence of the application of robonomics is the simple mechanism of commercialization and integration into the market. Among the advantages for business are: accurate task planning and performance evaluation, receiving feedback from sales and automatic correction of service behavior, filtering inefficient services, and reducing costs. ${ }^{32}$

In the following sections, we will present three concepts of robotic services organized according to these principles. 


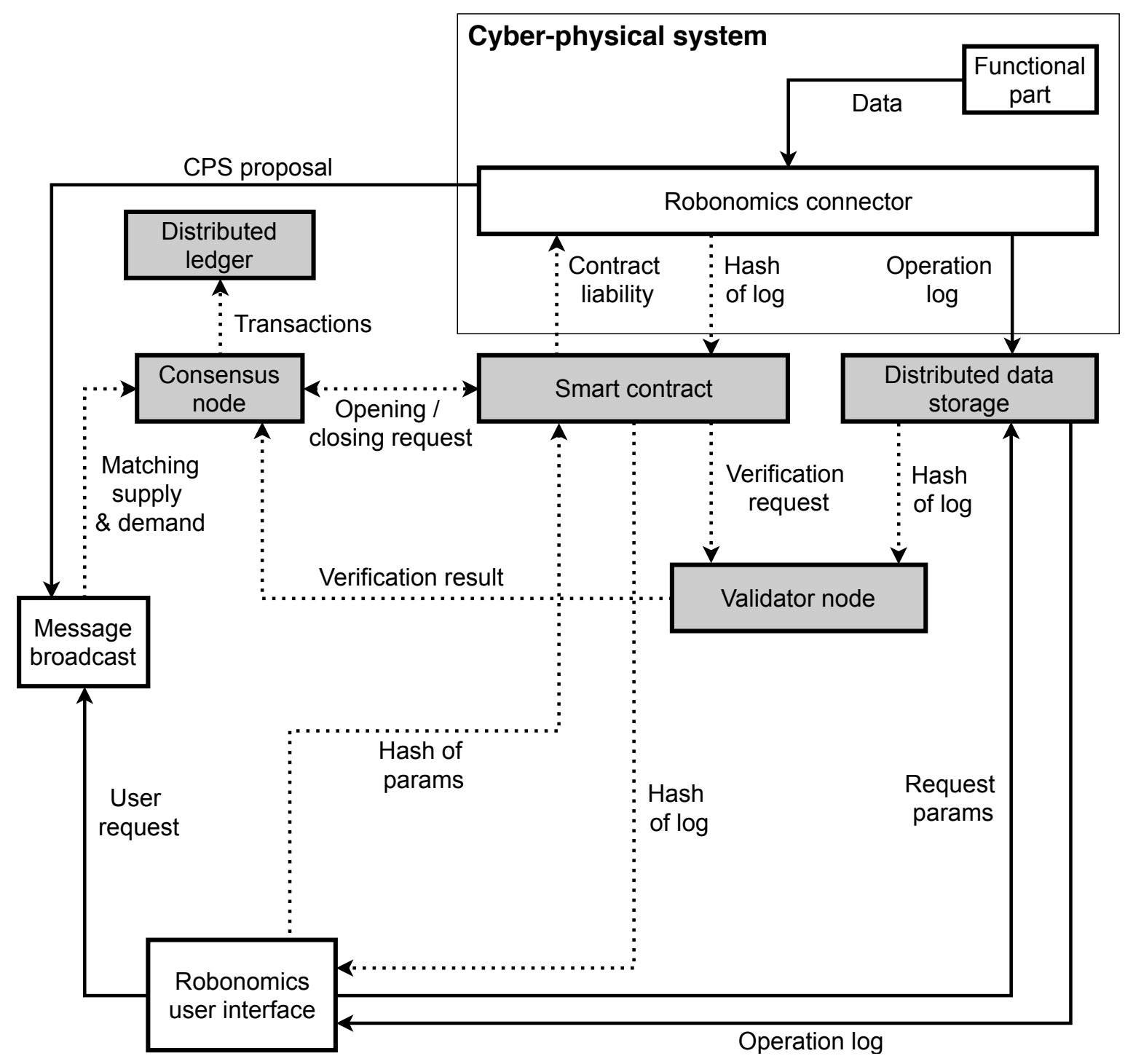

Fig. 1. Example of a robonomics network: gray blocks show the parts of a distributed network, dashed arrows show robonomics network information, and solid arrows show transfer of other information. The Consensus node sends economically meaningful robonomics data to the blockchain, if a supply and demand correspondence exists. The Validator node verifies the stated model of the contract terms with the model of the completed result and confirms its implementation.

\section{Unmanned Transport Systems}

An important element of the smart city infrastructure is a network of unmanned vehicles, to which land vehicles, aircraft, and watercraft are connected. ${ }^{33-35}$ In our opinion, the basic infrastructure of the network is as follows: traffic control system; transport optimization and logistics coordination system; stations servicing unmanned vehicles (automatic garage or base); order formation system that ensures the interaction of citizens with transport services; cargo handling stations; automatic cargo; and transport insurance services.

It can be seen that the coordination of the operation of the nodes requires considerable effort, and the issue of protection against errors, breaking and illegal actions must be resolved. We 
believe that the principles of robot economy are perfectly suited to the tasks of organizing a transport network. Each node publishes the demand for the services of other nodes, and forms its own offer. When the supply and demand match on the nodes, an agreement is made for the internal network tokens. The market mechanism of interaction makes it easier to optimize the transport network, create a unified mechanism for interaction between heterogeneous nodes and easily integrate communication with client-citizens (Fig. 2). In addition, the security and fault tolerance of the network is provided by decentralized technologies.

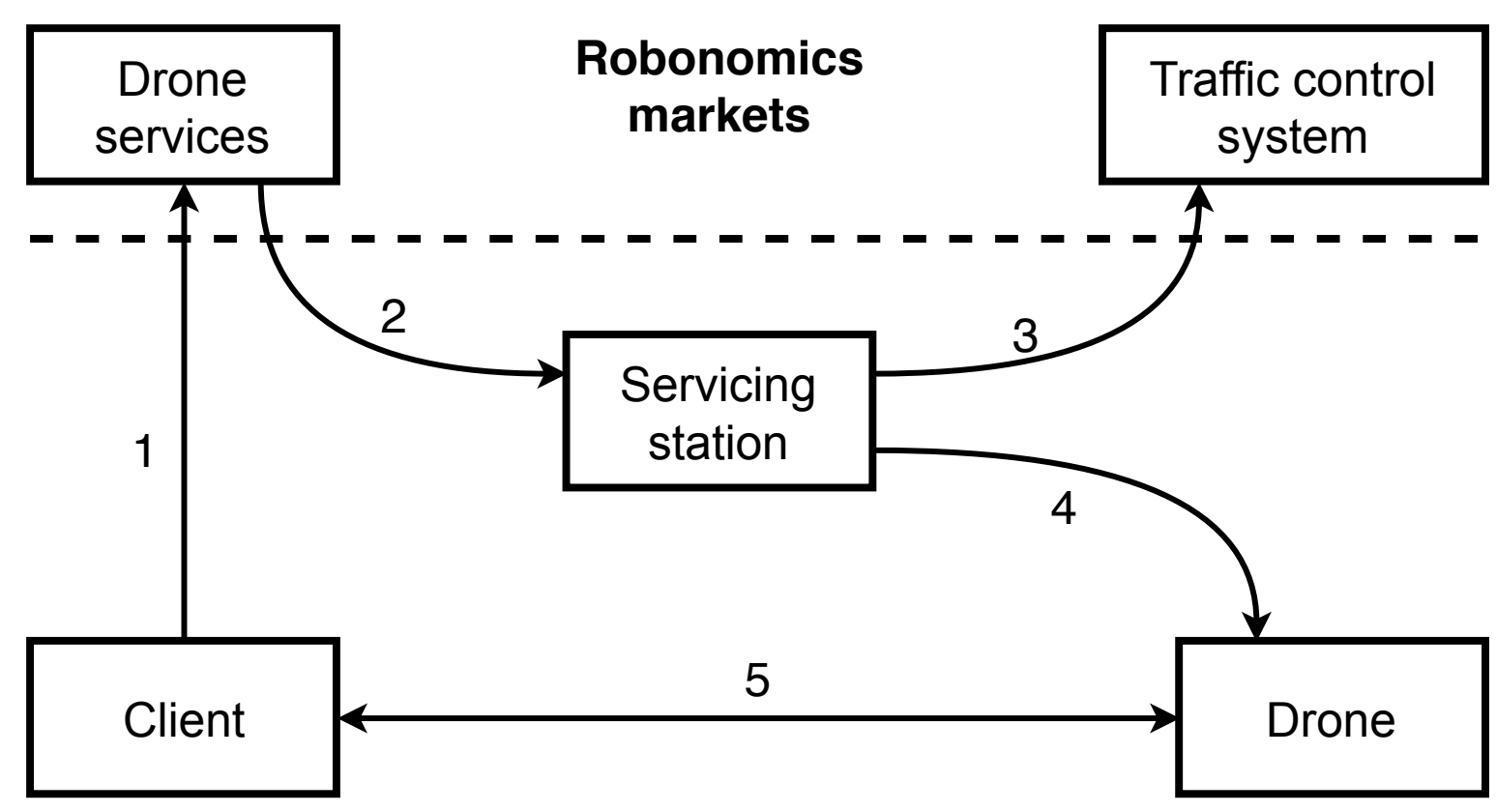

Fig. 2. Example of aircraft transport system functioning: 1) the client sends a request to markets; 2) the service with best offer wins the client; 3 ) the servicing station orders the route; 4) the station sends all paramaters of that flight to the drone; and 5) the smart contract is created between client and drone, and the client pays and receives a service

\section{Ecological Monitoring of the Environment}

A favorable ecological situation is an important possibility for smart cities. ${ }^{36}$ There are already solutions for creating environmental monitoring with the help of IoT devices, but the question remains how to organize this process in the most effective way. ${ }^{37}$

A promising way to protect the environment is to transform this sphere into a commercially attractive one. This is how the concept of carbon credits emerged. ${ }^{38}$ The essence of the concept is the issuance of purchase quotas to polluting companies for emissions of harmful substances. If the quota is exceeded, the company is fined, so the management has two choices: either to switch to more environmentally friendly production, or buy a new carbon credit.

Based on this mechanism, it is possible to organize a service for commercial monitoring and pollution control in a smart city. Citizens and companies are given the opportunity to freely connect mobile and stationary measuring devices to a common digital platform based on the robot economy. Similarly, services are connected to the network, which analyze the received 
data.

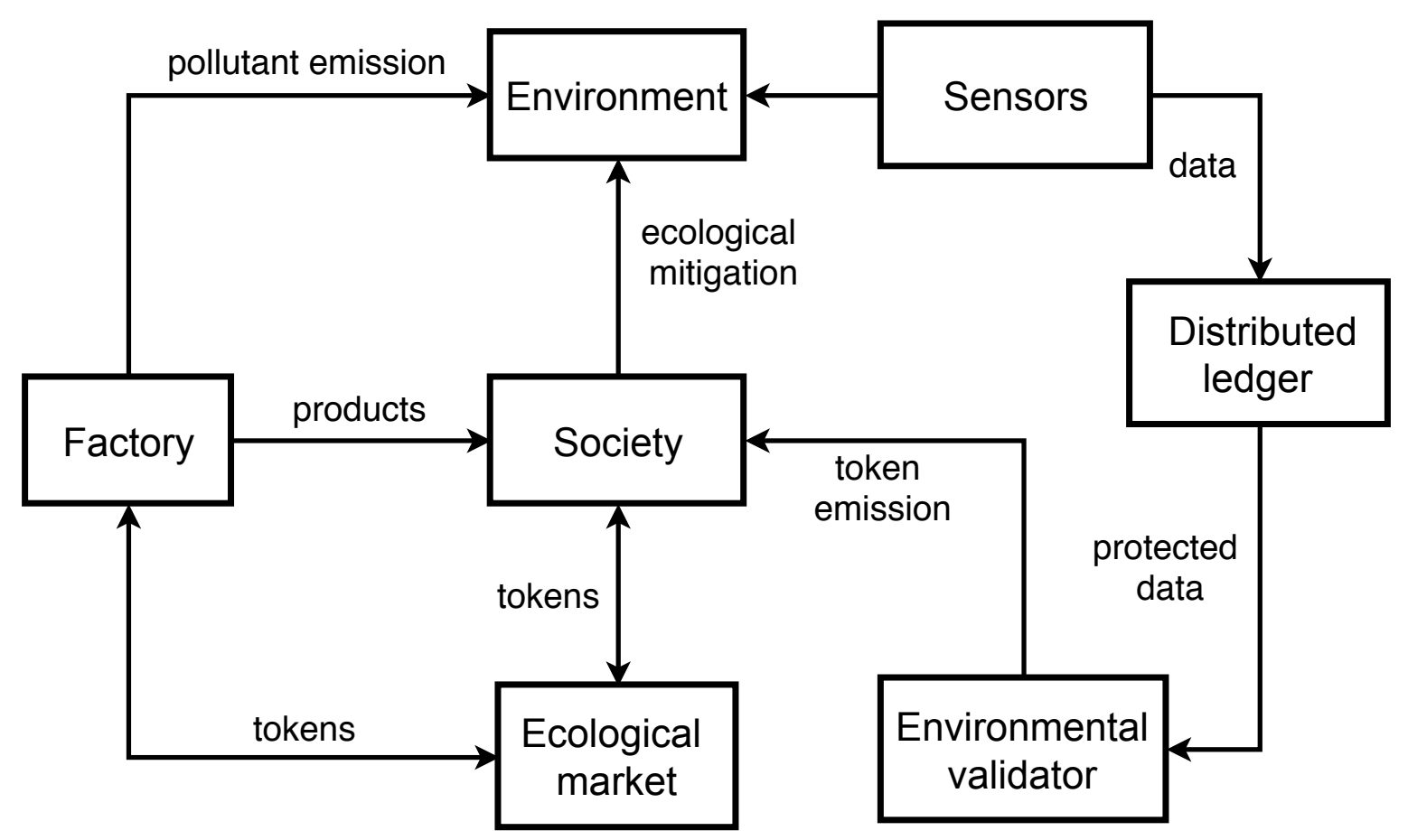

Fig. 3. Example of tokenization process through civil and commercial monitoring

This is how the automatic environmental inspection service is formed, which records all information about pollution in a blockchain. This information provides support for an internal token, which serves as a carbon credit available for exchange. Negative ecological externalities of a smart city are tokenized (Fig. 3), and citizens and city services are given the opportunity to control pollution through economic mechanisms.

\section{Supply Chain Management and Production as a Service}

An important component of a smart city should be smart factories, in which autonomous production occurs through the collection and exchange of information between people, materials and machine systems. ${ }^{39}$ Due to the large number of autonomous agents, the organization of a smart factory is subject to requirements to ensure flexibility of operation, scalability, quality of feedback, fault tolerance, and security. ${ }^{40}$ The principles of the robot economy and decentralized technologies make it possible to organize the factory in an effective way and solve these technological problems. In addition, the factory becomes responsive to demand, which is formed by citizens, and thereby becomes economically profitable.

For example (Fig. 4), the production of individual products for citizens is as follows. A citizen makes out an order for the manufacture of the product, applying a description by template or a detailed digital drawing. Having received the notification of payment, the service for processing requests makes the order validation. After validation, the factory management system forms a complete production process and directs it to the workshop. Then automatic production line 
produces the product. After checking the product, it is sent to the automatic warehouse, where it can be picked up by the customer or it can be transferred to the delivery service.

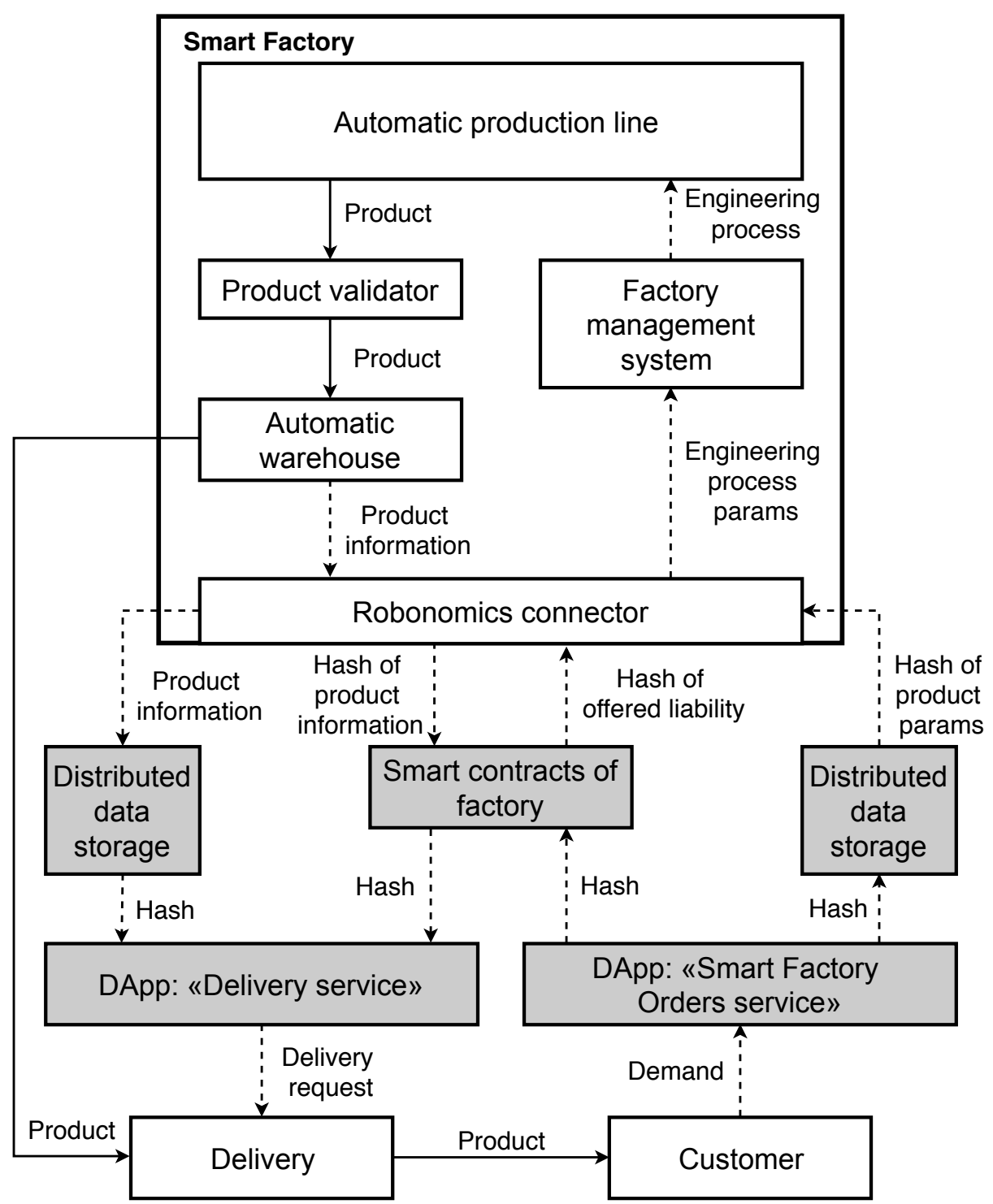

Fig. 4. Example of smart factory operation: gray blocks show the parts of distributed network, dashed arrows show information transfer, solid arrows show product transfer, decentralized application (DApp) software for interaction with decentralized networks.

In the case of mass production, factories must decide what type of goods should be produced, what resources should be procured and in what district of the city to distribute. Calculating operating costs and costs for market analysis, the factory can find the optimal way of production.

\section{Examples of Experiments}

The implementation of the described ideas is shown in this article. ${ }^{23}$ In the field of mobile robotics, software was developed for automatic control of a fleet of drones. ${ }^{41}$ In it the integration of ROS-compatible drones and the decentralized computer Ethereum was implemented. The 
developed methods are currently being expanded to the entire sphere of mobile robotics: for example, the methods have been successfully tested in an experiment on the organization of an autonomous taxi service. ${ }^{42}$

To confirm the efficiency of environmental monitoring based on the principles of robot economics, a successful experiment to create a network of sensors, that measure chemical performance, was conducted. ${ }^{43}$

To demonstrate the capabilities of smart factories, a laboratory stand was assembled simplifying the production and economic process (Fig. 5). ${ }^{44}$

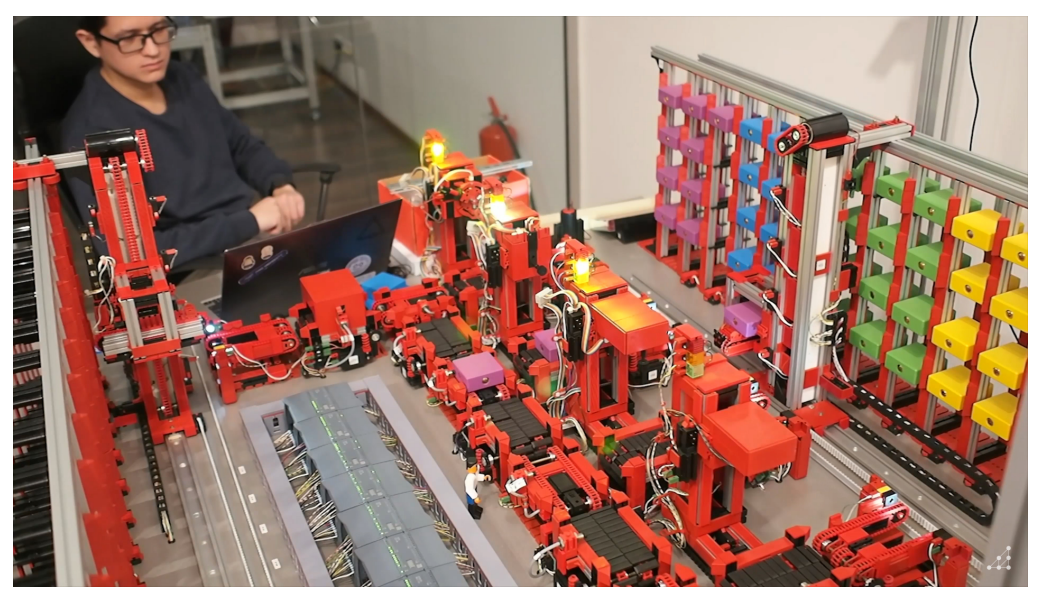

Fig. 5. Smart factory stand on the basis of Fischertechnik set and Siemens programmable controllers

The production "machines" of the stand reacted to changing capital on the markets of different types of goods and adjusted to them, trying to produce more products for those with higher capital.

\section{Conclusion}

We presented an example of an architectural solution for smart city management systems. Ethereum and ROS were proposed, as a mechanism of interaction-market mechanisms that allow to ensure the effectiveness and practicality of the agents. The article shows that the services of a smart city, are more efficiently and effectively managed when implemented based on the robot economy.

In the near future, the world will see an increase in the number of smart cities and the populations living in them, which will lead to an increase in the consumption of services that will be increasingly provided by robots, smart systems and artificial intelligence. The concept of a robot economy based on blockchain technologies, and in particular on the Ethereum network, may provide an excellent solution framework that combines security of communication, own data control by the citizens, potentially unlimited scalability, and unified standards of agent interaction within the network. Of course a number of technological, organizational and policy issues will need to be solved. 
LEDGER VOL 4, S1 (2019) 56-66

\section{Acknowledgement}

This work was financially supported by Government of Russian Federation (Grant 08-08). The work presented in this paper was partially supported by the ERASMUS+ Key Action 2 (Strategic Partnership) project IOT-OPEN.EU (Innovative Open Education on IoT: improving higher education for European digital global competitiveness), reference no.2016-1-PL01-KA203026471 .

This project has also received funding from the European Union's Horizon 2020 Research and Innovation Programme under Marie Skłodowska-Curie grant agreement No. 751615 (BROS). The European Commission support for the production of this publication does not constitute endorsement of the contents which reflects only the views of the authors, and the Commission cannot be held responsible for any use which may be made of the information contained therein.

\section{Notes and References}

\footnotetext{
${ }^{1}$ Schwandt, F. "Internet of Things (IoT) Connected Devices Installed Base Worldwide from 2015 to 2025 (in Billions)." Statista (2016) (accessed 10 September 2018) https://www.statista.com/statistics/ 471264/iot-number-of-connected-devices-worldwide/.

2 Parthasarathy, A. "Robotics Will Be Mainstream in 2017." The Week (2017) (accessed 10 September 2018) https://www.theweek.in/content/archival/news/sci-tech/Robotics-willbe-mainstream-in.html.

${ }^{3}$ Capdevila, I., Zarlenga, M. I. "Smart City or Smart Citizens? The Barcelona Case." Journal of Strategy and Management 8.3 266-282 (2015) https : //doi .org/10.1108/JSMA-03-2015-0030.

${ }^{4}$ Farag, A. A. "The Story of NEOM City: Opportunities and Challenges." In New Cities and Community Extensions in Egypt and the Middle East Cham: Springer 35-49 (2018).

${ }^{5}$ Cocchia, A. "Smart and Digital City: A Systematic Literature Review." In Smart City Cham: Springer $13-43$ (2014).

${ }^{6}$ Anthopoulos, L. G., Janssen, M., Weerakkody, V. "Comparing Smart Cities with Different Modeling Approaches." In WWW'15 Companion Proceedings of the 24th International Conference on World Wide Web New York: ACM 525-528 (2015) http://dx.doi.org/10.1145/2740908.2743920.
}

7 Angelidou, M. "Smart City Policies: A Spatial Approach.” Cities 41 S3-S11 (2014) https://doi .org/ 10.1016/j.cities.2014.06.007.

${ }^{8}$ Lin, H., Hu, J., Ma, J., Xu, L., Yu, Z. “A Secure Collaborative Spectrum Sensing Strategy in Cyber-Physical Systems.” IEEE Access 5 27679-27690 (2017) https : //doi .org/10.1109/ACCESS . 2017 . 2767701.

${ }^{9}$ Botta, A., De Donato, W., Persico, V., Pescapé, A. "Integration of Cloud Computing and Internet of Things: A Survey." Future Generation Computer Systems 56 684-700 (2016) https : //doi .org/10.1016/ j.future.2015.09.021.

${ }^{10}$ Minoli, D., Sohraby, K., Occhiogrosso, B. "IoT Considerations, Requirements, and Architectures for Smart Buildings-Energy Optimization and Next-Generation Building Management Systems.” IEEE Internet of Things Journal 4.1 269-283 (2017) https : //doi .org/10.1109/JIOT . 2017. 2647881.

${ }^{11}$ Lin, J., Yu, W., Zhang, N., Yang, X., Zhang, H., Zhao, W. "A Survey on Internet of Things: Architecture, Enabling Technologies, Security and Privacy, and Applications.” IEEE Internet of Things Journal 4.5 11251142 (2017) https: //doi .org/10.1109/JIOT . 2017.2683200.

${ }^{12}$ Castelló Ferrer, E., Rudovic, O., Hardjono, T., Pentland, A. "RoboChain: A Secure Data-Sharing Framework for Human-Robot Interaction.” arXiv (2018) (accessed 9 March 2019) http: //arxiv . org/abs/1802 . 04480 . 
${ }^{13}$ Waedt, K., Ciriello, A., Parekh, M., Bajramovic, E. "Automatic Assets Identification for Smart Cities: Prerequisites for Cybersecurity Risk Assessments." In 2016 IEEE International Smart Cities Conference (ISC2) 1-6 (2016) https://doi.org/10.1109/ISC2 .2016.7580812.

${ }^{14}$ Kshetri, N. "Can Blockchain Strengthen the Internet of Things?" IT Professional 19.4 68-72 (2017) https : //doi.org/10.1109/MITP.2017.3051335.

${ }^{15} \mathrm{Su}$, P. H., Shih, C.-S., Hsu, J. Y.-J., Lin, K.-J., Wang, Y.-C. "Decentralized Fault Tolerance Mechanism for Intelligent iot $/ \mathrm{m} 2 \mathrm{~m}$ Middleware." In 2014 IEEE World Forum on Internet of Things (WF-IoT) 45-50 (2014) https://doi.org/10.1109/WF-IoT.2014.6803115.

${ }^{16}$ Stoelen, M. F., de Tejada, V. F., Huete, A. J., Balaguer, C., Bonsignorio, F. P. "Distributed and Adaptive Shared Control Systems: Methodology for the Replication of Experiments." IEEE Robotics \& Automation Magazine 22.4 137-146 (2015) https : //doi . org/10.1109/MRA . 2015.2460911.

${ }^{17}$ Skarmeta, A. F., Hernandez-Ramos, J. L., Moreno, M. V. “A Decentralized Approach for Security and Privacy Challenges in the Internet of Things." In 2014 IEEE World Forum on Internet of Things (WF-IoT) 67-72 (2014) https://doi .org/10.1109/WF-IoT . 2014.6803122.

${ }^{18}$ Xu, X., et al. "The Blockchain as a Software Connector." In 2016 13th Working IEEE/IFIP Conference on Software Architecture (WICSA) 182-191 (2016) https://doi .org/10.1109/WICSA . 2016.21.

${ }^{19}$ Castelló Ferrer, E. “The Blockchain: A New Framework for Robotic Swarm Systems.” In Proceedings of the Future Technologies Conference (FTC) 2018 Cham: Springer 1037-1058 (2018) https://dx.doi .org/ 10.1007/978-3-030-02683-7_77.

${ }^{20}$ Wohrer, M., Zdun, U. "Smart Contracts: Security Patterns in the Ethereum Ecosystem and Solidity." In 2018 International Workshop on Blockchain Oriented Software Engineering (IWBOSE) 2-8 (2018) https: //doi.org/10.1109/IWBOSE.2018.8327565.

${ }^{21}$ Strobel, V., Castelló Ferrer, E., Dorigo, M. "Managing Byzantine Robots via Blockchain Technology in a Swarm Robotics Collective Decision Making Scenario." In AAMAS '18 Proceedings of the 17th International Conference on Autonomous Agents and MultiAgent Systems Richland: International Foundation for Autonomous Agents and Multiagent Systems 541-549(2018) https://dl.acm.org/citation.cfm?id= 3237464.

${ }^{22}$ Norta, A. "Creation of Smart-Contracting Collaborations for Decentralized Autonomous Organizations." In International Conference on Business Informatics Research Cham: Springer 3-17 (2015) https://doi. org/10.1007/978-3-319-21915-8_1.

${ }^{23}$ Lonshakov, S., Krupenkin, A., Kapitonov, A., Radchenko, E., Khassanov, A., Starostin, A. "Robonomics: Platform for Integration of Cyber Physical Systems into Human Economy." Robonomics (2018) (accessed 10 September 2018) https://goo.gl/pNH8wK.

${ }^{24}$ Bigi, G., Bracciali, A., Meacci, G., Tuosto, E. "Validation of Decentralised Smart Contracts through Game Theory and Formal Methods." In Programming Languages with Applications to Biology and Security Cham: Springer 142-161 (2015)https://doi.org/10.1007/978-3-319-25527-9_11.

${ }^{25}$ Harz, D., Boman, M. “The Scalability of Trustless Trust.” arXiv (2018) (accessed 9 March 2019) http: //arxiv.org/abs/1801.09535.

${ }^{26}$ Quigley, M., et al. "ROS: An Open-Source Robot Operating System." In ICRA Workshop on Open Source Software 1-5 (2009) http://www.willowgarage.com/papers/ros-open-source-robot-operatingsystem.

${ }^{27}$ Chauhan, A., Malviya, O. P., Verma, M., Mor, T. S. "Blockchain and Scalability." In 2018 IEEE International Conference on Software Quality, Reliability and Security Companion (QRS-C) 122-128 (2018) https://doi.org/10.1109/QRS-C.2018.00034.

${ }^{28}$ Hertig, A. "How Will Ethereum Scale?" Coindesk (2018) (accessed 10 September 2018) https://www . coindesk.com/information/will-ethereum-scale/.

${ }^{29}$ Li, W., Andreina, S., Bohli, J.-M., Karame, G. "Securing Proof-of-Stake Blockchain Protocols.” In Data Privacy Management, Cryptocurrencies and Blockchain Technology Cham: Springer 297-315 (2017) https: //doi.org/10.1007/978-3-319-67816-0_17. 
${ }^{30}$ Iyer, K., Dannen, C. "Crypto-Economics and Game Theory." In Building Games with Ethereum Smart Contracts Berkeley: Apress 129-141 (2018).

${ }^{31}$ Kim, J. "Vitalik Buterin: Sharding and Plasma to Help Ethereum Reach 1 Million Transactions Per Second." Cryptoslate (2018) (accessed 10 September 2018) https://goo.gl/pXWHCc.

${ }^{32}$ Davidson, S., De Filippi, P., Potts, J. "Economics of Blockchain.” (2016) (accessed 9 March 2019) https : //doi.org/10.2139/ssrn.2744751.

${ }^{33}$ Zhang, X., Gao, H., Guo, M., Li, G., Liu, Y., Li, D. “A Study on Key Technologies of Unmanned Driving.” CAAI Transactions on Intelligence Technology 1.1 4-13 (2016) https:/doi.org/10.1016/j.trit. 2016. 03.003.

${ }^{34}$ Valavanis, K. P., Vachtsevanos, G. J. "Future of Unmanned Aviation." In Handbook of Unmanned Aerial Vehicles Dordrecht: Springer 2993-3009 (2015).

${ }^{35}$ Shuo, J., Yonghui, Z., Wen, R., Kebin, T. "The Unmanned Autonomous Cruise Ship for Water Quality Monitoring and Sampling." In 2017 International Conference on Computer Systems, Electronics and Control (ICCSEC) 700-703 (2017) https:/doi.org/10.1109/ICCSEC.2017.8447040.

${ }^{36}$ Monzon, A. "Smart Cities Concept and Challenges: Bases for the Assessment of Smart City Projects." In 2015 International Conference on Smart Cities and Green ICT Systems (SMARTGREENS) 1-11 (2015) https://ieeexplore.iee.org/abstract/document/7297938.

${ }^{37}$ Jamil, M. S., Jamil, M. A., Mazhar, A., Ikram, A., Ahmed, A., Munawar, U. “Smart Environment Monitoring System by Employing Wireless Sensor Networks on Vehicles for Pollution Free Smart Cities." Procedia Engineering 107 480-484 (2015) https://doi.org/10.1016/j .proeng. 2015.06.106.

${ }^{38}$ Agrawal, S., Tiwari, G. N. "Performance Analysis in Terms of Carbon Credit Earned on Annualized Uniform Cost of Glazed Hybrid Photovoltaic Thermal Air Collector." Solar Energy 115 329-340 (2015) https://doi.org/10.1016/j.solener.2015.02.030.

${ }^{39}$ Radziwon, A., Bilberg, A., Bogers, M., Madsen, E. S. "The Smart Factory: Exploring Adaptive and Flexible Manufacturing Solutions." Procedia Engineering 69 1184-1190 (2014) https://doi.org/10.1016/j. proeng.2014.03.108.

${ }^{40}$ Leitão, P., Karnouskos, S. (Eds.) Industrial Agents: Emerging Applications of Software Agents in Industry. Burlington: Morgan Kaufmann (2015).

${ }^{41}$ Kapitonov, A., Lonshakov, S., Krupenkin, A., Berman, I. "Blockchain-Based Protocol of Autonomous Business Activity for Multi-Agent Systems Consisting of UAVs." In 2017 Workshop on Research, Education and Development of Unmanned Aerial Systems (RED-UAS) 84-89 (2017) https://doi.org/10.1109/REDUAS . 2017.8101648.

42 Krupenkin, A. “Airalab - Duckietown Taxi Service.” Medium (2017) (accessed 10 September 2018) https : //blog.aira.life/duckietown-taxi-service-db192f4ff6cc.

${ }^{43}$ Bulatov, V. "Consensus 2018 Hackathon: Economically Independent Sensor Networks Built in a Day." Medium (2018) (accessed 10 September 2018) https://goo.gl/YyBtCs.

${ }^{44}$ Khassanov, A. "Testing the Game Robonomics Based on the Model Set Fischertechnik Controlled by PLC Siemens." Youtube (2018) (accessed 10 September 2018) https://www.youtube.com/watch?v=Lj IB34kQOA.

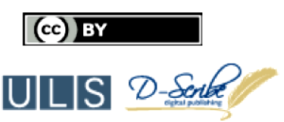

Articles in this journal are licensed under a Creative Commons Attribution 4.0 License.

Ledger is published by the University Library System of the University of Pittsburgh as part of its D-Scribe Digital Publishing Program and is cosponsored by the University of Pittsburgh Press. 